\title{
Interventional Neuroradiology on Call: The Need for Emergency Coiling of Ruptured Intracranial Aneurysms
}

S

ubarachnoid hemorrhage is a serious disease with a case fatality of approximately $50 \%$. Aneurysms are the cause of SAH in $85 \%$ of cases. Rebleeding is the most imminent concern; therefore, the first aim is occlusion of the ruptured aneurysm. ${ }^{1}$

In our institution with 150-200 SAHs annually, 3 interventional neuroradiologists, and 13 neurosurgeons (3 with vascular expertise), the diagnostic and therapeutic logistics to achieve this goal are as follows: Patients with SAH are admitted to the emergency department by neurologic or neurosurgical residents. Most patients come from outside hospitals. After stabilization, native brain CT and CT angiography are performed, followed by angiography on short notice during office hours. In poor-grade or intubated patients, angiography is performed with the patient under general anesthesia. If an aneurysm is found, coiling is the first treatment option. If coiling seems technically difficult, neuroradiologic and neurosurgical colleagues are consulted about the best treatment (coiling, balloon- or stent-assisted coiling, parent vessel occlusion, or clipping) and the best treating physician for this particular patient. Subsequently, coiling is performed with the patient under general anesthesia immediately following angiography, or clipping is scheduled for the next day in good-grade patients and is postponed in poor-grade patients. In the past 2 decades, since the introduction of coiling, this diagnostic and therapeutic pathway has been firmly established and all patients with ruptured aneurysms who are admitted on weekdays are coiled within 24 hours.

On the basis of adequate logistics, a general perception of caretakers in the hospital (emergency department, intensive care, neurodivision) is that a patient with an SAH should be coiled immediately; if not, he or she will probably die from a rebleed. For the weekend, this implies that whenever a patient with SAH is admitted, the interventional neuroradiologist is consulted immediately for diagnosis and treatment. However, during the weekend, the optimal logistics of weekdays cannot always be met. Anesthesia is difficult to get, deliberation with radiologic and neurosurgical colleagues about the most appropriate treatment is usually not possible, experienced technicians may not be on call, and the mindset of the operating team may not be optimal because of private circumstances. At that time, it is the individual

http://dx.doi.org/10.3174/ajnr.A4134 decision of the interventional neuroradiologist whether to treat and with which technique. When complications occur, there is no help at hand. Therefore, during the weekend, the chances of making a wrong decision that negatively affects outcome is higher than on weekdays.

The following case may be instructive: A 20-year-old medical student experienced sudden severe headache early Sunday morning. CT in another hospital showed an SAH (Fig 1A), and the patient was transferred to our institution, arriving at noon. CT angiography demonstrated a vertebral junction aneurysm (Fig $1 B$ ). The neurosurgeon on call requested early coiling. However, at that time, anesthesia in the angiography suite was impossible because of busy operating rooms. Because the patient became gradually drowsy from a developing hydrocephalus, it was decided to place an external ventricular drain in the afternoon and postpone coiling until Monday morning. However, the caretakers in the hospital had informed the patient's family about an imminent rebleed and had promised coiling that very day. Therefore, the family insisted compellingly on urgent coiling. Because the anesthesia team was too busy for coiling that afternoon, it was finally decided to do the coiling procedure directly following the ventricular drainage; the patient was to be transported intubated and sedated from the operating room. In the angiography suite, the intensivist would take care of deep sedation during the coiling procedure. When the patient arrived intubated and sedated in the angiography suite late in the afternoon, she was lifted onto the angiography table. At that moment, she regained consciousness and started to cough heavily while forcefully trying to remove the endotracheal tube. After several attempts, she suddenly dropped back on the table, returning comatose with bradycardia and hypertension. Apparently, the stress from the tube resulted in sudden hypertension and recurrent hemorrhage. Some 15 minutes later, 3D rotational angiography of the right vertebral artery demonstrated a vertebral junction aneurysm that was still bleeding (Fig 1C). After coiling, the bleeding had stopped, and the aneurysm was completely occluded. Her clinical course was complicated by drain-dependent hydrocephalus, vasospasm, and meningitis. Six months later, she is in a rehabilitation center with severe cognitive impairment, hemiparesis, untreatable central emesis, and bilateral abducens paresis.

This case illustrates that whenever the necessary diagnostic 

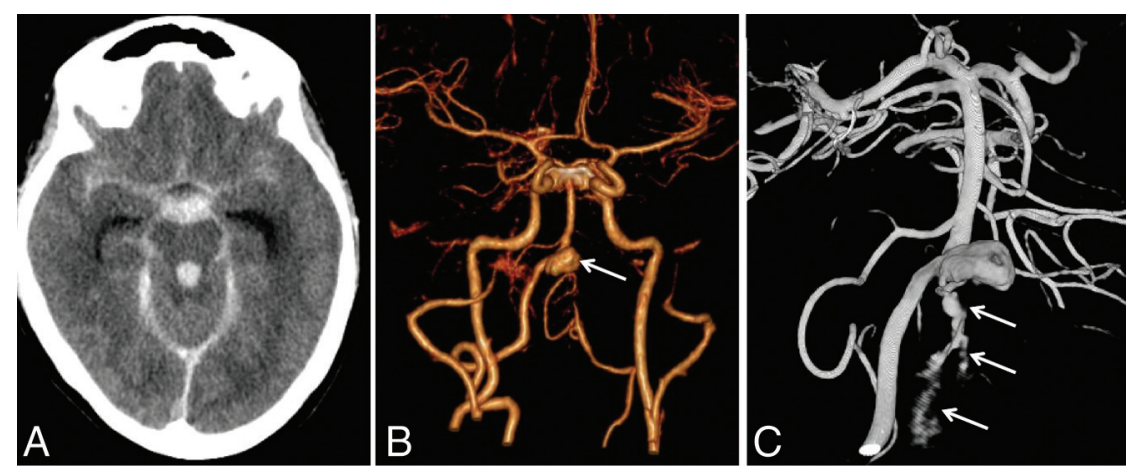

FIG 1. A 20-year-old patient with sudden headache. A, CT demonstrates diffuse SAH with beginning hydrocephalus. $B, C T A$ reveals a large vertebral junction aneurysm (arrow). C, 3D angiography with contrast extravasation from the aneurysm (arrows).

and therapeutic logistics are not completely available, there is room for wrong decisions and mistakes that can easily result in poor outcome for the patient. The perception of a high chance of rebleed by both caretakers and family compelled the treating team physicians to make the wrong decision: The instigated treatment strategy induced a rebleed instead of preventing it. It would have been better to postpone the coiling to the next day.

Coiling of ruptured aneurysms has become the technique of choice during the past 2 decades, and early treatment within 24 hours is now possible in all patients, regardless of clinical condition. In the clipping era, treatment was considered early within 72 hours, and patients in poor clinical condition often underwent an operation in a delayed fashion 2 weeks later. Although early coiling is possible, it is questionable whether it should be performed in all patients presenting with $\mathrm{SAH}$, given the higher risk of complications by wrong decision-making outside office hours. The magnitude of this higher risk because of impaired logistics is unknown and will vary from clinic to clinic. On the other hand, what is the additional risk to a patient with SAH when the coiling is postponed 1 or 2 days? What is the frequency of rebleed and how are rebleedings distributed over time after SAH?

When the aneurysm is left untreated, it is assumed that 30\%$40 \%$ of patients with SAH from a ruptured aneurysm will have a rebleed in the first 30 days after SAH. ${ }^{1}$ In a study by Ohkuma, ${ }^{2} 37$ of $273(14 \%)$ patients had a rebleed within the first 8 hours after aneurysm rupture, $30(81 \%)$ in the first 2 hours, mostly before admission to a hospital. After this early period, the distribution of rebleeds with time remains constant during the next 30 days. This rebleed risk from day 0.5 to day 30 is on the order of $1 \%$ per day. ${ }^{1-3}$ In practice, in virtually all patients with a ruptured aneurysm, the
6- to 8-hour period with the highest rebleed risk has passed at the time of intended treatment. Thus, an estimated rebleed risk of $1 \%$ per day is an accurate rule of thumb for daily practice.

Apart from this low risk of rebleed, early coil treatment is not relevant or not indicated in several subgroups of patients with SAH with the following conditions: a perimesencephalic bleeding pattern; an aneurysmal SAH pattern but negative CT angiography findings; SAH from AVMs or dural fistulas, moribund clinical condition; high age; trauma and $\mathrm{SAH}$; serious comorbidity; and a diagnostic delay of several days. These groups account for $>50 \%$ in our practice. In addition, after $3 \mathrm{D}$ angiography, in approximately $5 \%-10 \%$ of patients, it becomes clear that clipping is a better option than coiling. Thus, only a minority of patients with SAH might potentially benefit from early coil treatment.

In summary, a patient with a ruptured aneurysm has the highest risk of rebleed in the first few hours after rupture, mostly before admission to the hospital. After admission, the risk of rebleed is approximately $1 \%$ per day. In most patients with acute $\mathrm{SAH}$, early treatment needs no consideration. A minority of patients with SAH might potentially benefit from early coiling. However, when logistics on the weekend are different from those on weekdays, the small additional risk of rebleed when coiling is postponed probably outweighs the increased risk of complications by impaired logistics. In most institutions, including ours, a patient with acute SAH will be better off when not considered an emergency case.

\section{REFERENCES}

1. Van Gijn J, Kerr RS, Rinkel GJ. Subarachnoid hemorrhage. Lancet 2007;369:306-18

2. Ohkuma H, Tsurutani H, Suzuki S. Incidence and significance of early aneurysmal rebleeding before neurosurgical or neurological management. Stroke 2001;32:1176-80

3. Brilstra EH, Rinkel GJ, Algra A, et al. Rebleeding, secondary ischemia, and timing of operation in patients with subarachnoid hemorrhage. Neurology 2000;55:1656-60

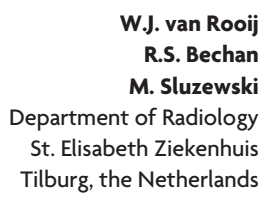

